\title{
Loss of ALDH1A1 expression is an early event in the pathogenesis of ovarian high-grade serous carcinoma
}

\author{
M Herman Chui ${ }^{1,2,5}$, Yihong Wang ${ }^{3,4,5}$, Ren-Chin $W^{1}$, Jeffrey Seidman ${ }^{1}$, \\ Robert J Kurman ${ }^{1,4}$, Tian-Li Wang ${ }^{1}$ and Ie-Ming Shih ${ }^{1,4}$ \\ ${ }^{1}$ Department of Pathology, Johns Hopkins Medical Institutions, Baltimore, MD, USA; ${ }^{2}$ Department of Laboratory \\ Medicine and Pathobiology, Faculty of Medicine, University of Toronto, Toronto, ON, Canada; ${ }^{3}$ Department of \\ Pathology, Sir Run Run Shaw Hospital, Zhejiang University School of Medicine, Hangzhou, China and \\ ${ }^{4}$ Department of Gynecology and Obstetrics, Johns Hopkins Medical Institutions, Baltimore, MD, USA
}

\begin{abstract}
Tumor-initiating cells are thought to share features with normal somatic stem cells. In mice, stem cells at the ovarian hilum have been shown to express the stem cell marker, aldehyde dehydrogenase isoform 1A1 (ALDH1A1), and are prone to malignant transformation. The potential relevance of this finding to humans has not been established. In this study, we used immunohistochemistry to assess the distribution of ALDH1A1 staining in the epithelium of human fallopian tubes, with particular reference to the transition of tubal epithelium to mesothelium (ie, tubal-mesothelial junction), ovarian surface epithelium, as well as putative precursors of ovarian high-grade serous carcinoma, namely, serous tubal intraepithelial carcinoma and ' $p 53$ signatures,' and overt serous carcinoma. Expression of ALDH1A1 was detected in both secretory and ciliated tubal epithelial cells, tubal-mesothelial junctions and ovarian surface epithelium, but was absent in serous tubal intraepithelial carcinoma and p53 signatures. Positive staining in high-grade serous carcinoma, when present, was typically limited to rare tumor cells. In silico analyses of the mRNA expression data set from The Cancer Genome Atlas revealed downregulation of ALDH1A1 transcripts in high-grade serous carcinoma relative to normal tubal epithelium, and no association between ALDH1A1 expression levels and overall survival. Our results do not support ALDH1A1 as a specific marker of stem cells in human fallopian tube and demonstrate that its loss of expression is an early event in the development of high-grade serous carcinoma.

Modern Pathology (2015) 28, 437-445; doi:10.1038/modpathol.2014.89; published online 12 September 2014
\end{abstract}

\section{Introduction}

Mounting evidence has shown that a significant proportion of pelvic high-grade serous carcinomas arise from a noninvasive occult carcinoma in the distal fallopian tube, designated 'serous tubal intraepithelial carcinoma'. ${ }^{1}$ These lesions have been identified in a small percentage of prophylactic salpingo-oophorectomy specimens from women with germline $B R C A$ mutations,,$^{2,3}$ and in the tubal fimbriae from up to $60 \%$ of women with sporadic ovarian serous carcinomas. ${ }^{4}$ Complete microscopic

Correspondence: Dr I-M Shih, MD, PhD, Department of Gynecology and Obstetrics, Johns Hopkins Medical Institutions, Cancer Research Building II, Room 305, 1550 Orleans Street, Baltimore, MD 212031, USA.

E-mail: ishih@jhmi.edu

${ }^{5} \mathrm{MHC}$ and $\mathrm{YW}$ are joint first authors and have contributed equally to this work.

Received 3 December 2013; revised 17 April 2014; accepted 9 May 2014; published online 12 September 2014 sectioning of fallopian tubes has also revealed focal proliferations of morphologically normal secretory cells that show diffuse TP53 immunoreactivity, termed 'p53 signatures, 5 which may represent early clonal expansions of tumor-initiating cells.

Experimental work conducted in a variety of model systems across different tumor types have consistently demonstrated that tumor-initiating cells are derived from either a somatic stem cell or an early progenitor cell that has reacquired stem cell features. ${ }^{6}$ This is in keeping with the concept that the capacity for indefinite proliferation, or selfrenewal, is essential for malignant transformation. ${ }^{7}$ Studies have also shown that only a fraction of cancer cells, termed 'cancer stem cells' are capable of forming tumors upon serial transplantation into immunocompromised mice, a technique used to assess self-renewal. ${ }^{8}$

One of the most commonly used markers for both normal and malignant stem cells is aldehyde dehydrogenase (ALDH) isoform 1A1 (ALDH1A1; 
previously known as ALDH1). ALDH1A1 is an enzyme involved in the metabolism of retinoic acid, which has been implicated in the regulation of cellular differentiation. ${ }^{9,10}$ Multipotent stem cells in normal tissues have been shown to express ALDH1A1, ${ }^{10,11}$ which has also been studied extensively as a candidate marker for cancer stem cells. ${ }^{11-15}$ In mice, the 'ovarian hilum,' where nerves and blood vessels enter the ovary, is covered by a layer of cells that have been previously described as representing the transition between mesothelium lining the surface of the ovary and ovarian bursa, and ciliated columnar epithelium lining the mouse oviduct. ${ }^{16}$ This region has been reported to be enriched for ALDH1A1-positive stem cells that show increased susceptibility to malignant transformation. ${ }^{16}$

In humans, the mesothelial lining of the ovarian surface epithelium extends over the ovarian hilum and is continuous with the mesovarium and broad ligament. The point of contact between mesothelium and Müllerian epithelium is positioned at multiple foci within the human fallopian tube fimbria and these 'tubal-mesothelial junctions' may be analogous to the junctional epithelium overlying the mouse ovarian hilum. ${ }^{17}$ Whether stem cells reside in either the hilar area or the tubal-mesothelial junctions in humans, as observed in the mouse model, however, remains unknown.

To gain further insight into the phenotype of the cell of origin and the early events in the pathogenesis of high-grade serous carcinoma, we used immunohistochemistry to examine the pattern of ALDH1A1 expression in normal and lesional fallopian tube epithelium, including tubal-mesothelial junctions, endosalpingiosis, p53 signatures, serous tubal intraepithelial carcinoma, and high-grade serous carcinoma, as well as in ovarian surface epithelium, rete ovarii, and primary and recurrent ovarian serous carcinomas.

\section{Materials and methods}

\section{Case selection}

Formalin-fixed paraffin-embedded surgical specimens were retrieved from the archives of the Department of Pathology, The Johns Hopkins Medical Institutions. Salpingectomy specimens were obtained from 46 patients: 29 normal fallopian tubes (with adjacent ovary and intact ovarian surface epithelium in 8 cases) and 17 with serous tubal intraepithelial carcinoma. Within these specimens, 28 tubal-mesothelial junctions, 14 foci of endosalpingiosis, 11 p53 signatures, and 11 overt serous carcinomas were identified. Further analyses were performed on a tissue microarray comprising 28 pairs of matched primary and recurrent ovarian high-grade serous carcinomas.

Diagnoses of serous tubal intraepithelial carcinoma and p53 signatures were made according to previously reported criteria. ${ }^{18,19}$ Tubal-mesothelial junctions were identified based on morphological features, with questionable foci confirmed by immunohistochemistry for calretinin, as previously described. ${ }^{17}$ The acquisition of tissue samples was approved by the institutional review board.

\section{Western blot analysis}

Western blot analysis was performed on the HHL-6 human hepatocyte cell line, and protein lysates were separated by electrophoresis on $4-12 \%$ Trisglycine gels and transferred onto a polyvinylidine difluoride membrane. After being blocked with 5\% non-fat milk, the membrane was incubated at $4{ }^{\circ} \mathrm{C}$ overnight with two separate ALDH1A1 primary antibodies (EP1932Y, Epitomics and clone 44, BD Biosciences), both at 1:500 dilution, and washed, incubated with horseradish peroxidase-conjugated secondary antibody, and detected with ECL detection reagent (GE Healthcare).

\section{Immunohistochemistry}

The following primary antibodies were used: TP53 mouse monoclonal (clone Bp53-11, Ventana), Pax-8 rabbit polyclonal (Proteintech), calretinin rabbit polyclonal (Invitrogen), ALDH1A1 rabbit monoclonal (clone EP1932Y, Epitomics), and ALDH1A1 mouse monoclonal (clone 44, BD Biosciences). Details of optimized reaction conditions are summarized in Table 1.

Of note, we evaluated two separate antibodies against ALDH1A1. Antigen retrieval was performed by steaming the sections in citrate buffer ( $\mathrm{pH} \mathrm{6.0)}$ for 25 min. Blocking of endogenous peroxidases was performed by immersing slides in 3\% hydrogen peroxide for $15 \mathrm{~min}$, followed by blocking using an

Table 1 Immunohistochemical staining details

\begin{tabular}{|c|c|c|c|c|c|}
\hline Markers & Source & Clone & Species & Retrieval & Dilution \\
\hline TP53 & Ventana & Bp53-11 & Mouse & Citrate (pH 6.0) & 1:1 (Pre-diluted) \\
\hline Calretinin & Invitrogen & Polyclonal & Rabbit & Citrate (pH 6.0) & 1:1 (Pre-diluted) \\
\hline Pax-8 & Proteintech & Polyclonal & Rabbit & Citrate (pH 6.0) & $1: 2000$ \\
\hline \multirow[t]{2}{*}{ ALDH1A1 } & Epitomics & EP1932Y & Rabbit & Citrate (pH 6.0) & $1: 300$ \\
\hline & BD Biosciences & 44 & Mouse & Citrate (pH 6.0) & $1: 600$ \\
\hline
\end{tabular}


avidin/biotin blocking kit (Invitrogen), according to the manufacturer's instructions. Slides were incubated with $5 \%$ non-fat milk for $30 \mathrm{~min}$ at room temperature and overnight at $4{ }^{\circ} \mathrm{C}$ with ALDH1A1 antibody at 1:300 dilution (EP1932Y) or 1:600 dilution (clone 44). Following wash steps, slides were incubated with peroxidase-conjugated polyclonal goat anti-rabbit antibody (Dako, for EP1932Y) or polyclonal rabbit anti-mouse antibody (Jackson ImmunoResearch, for clone 44), and visualized using 3,3'-diaminobenzidene.

Confirmation of appropriate ALDH1A1 staining was performed on control slides of normal colonic and endometrial tissue. The pattern of ALDH1A1 staining was analyzed semi-quantitatively in normal tubal epithelium, ovarian surface epithelium, p53 signatures, serous tubal intraepithelial carcinoma, endosalpingiosis, rete ovarii, and serous carcinoma, subsequent to identification of areas of interest on corresponding hematoxylin and eosin- and TP53stained slides. Lesions were considered positive for ALDH1A1 if any of the cells showed strong definitive cytoplasmic staining. In p53 signatures, endosalpingiosis, rete ovarii, and tubal intraepithelial carcinomas, the percentage of ALDH1A1-positive epithelial cells was estimated by counting all observable cells, whereas for tubal-mesothelial junctions, at least 10 cells flanking each side of the junction (ie, $\geq 20$ consecutive cells in total) were counted. For each sample of normal fallopian tube and carcinoma, at least 500 epithelial cells were assessed. Evaluation of ovarian surface epithelium was limited to eight ovaries with intact surface epithelium spatially distributed across different regions (ie, hilum, within cortical clefts, other). Immunohistochemical staining for Pax-8 and calretinin was performed to assess whether columnar cells lining the ovarian surface were of mesothelial or tubal derivation.

ALDH1A1 staining was also assessed on tissue microarrays comprising 26 pairs of primary and matched recurrent high-grade ovarian serous carci- nomas. The percentage of ALDH1A1-positive tumor cells was estimated for each core on the tissue microarray and averaged across triplicates. Mean percentages of ALDH1A1-positive cells were compared between groups using the paired $t$-test (twotailed). Of note, all samples in this study were stained with the ALDH1A1 antibody clone EP1932Y. Confirmation of staining results was subsequently carried out using the clone 44 antibody on control tissues, tissue microarray sections, and a subset of serous tubal intraepithelial carcinomas.

\section{In silico analyses}

Level 3 Agilent $244 \mathrm{~K}$ custom gene expression G4502A-07 platform mRNA microarray data were downloaded from the TCGA data portal (https://tcgadata.nci.nih.gov/tcga/, 31/9/2013), which included 558 primary ovarian serous carcinomas with available clinical data, 8 normal fallopian tube controls and 15 recurrent ovarian serous carcinomas. The unpaired Welch 2-sample $t$-test was performed to compare ALDH1A1 expression between 558 primary tumors and 8 normal (fallopian tubes) samples. Overall survival was compared in patients with ALDH1A1high and ALDH1A1-low tumors (above and below median expression level, respectively) by KaplanMeir analysis and log-rank test. Primary and recurrent tumors were compared using the paired $t$-test.

\section{Results}

For both ALDH1A1 antibodies, obtained from Epitomics and BD Biosciences, western blotting revealed a robust band at $55 \mathrm{kDa}$, corresponding to the molecular weight of ALDH1A1 (Figure 1a). This result confirmed the specificity of both antibodies in recognizing the ALDH1A1 protein. Immunostaining using both antibodies demonstrated that the positively stained cells were largely restricted to
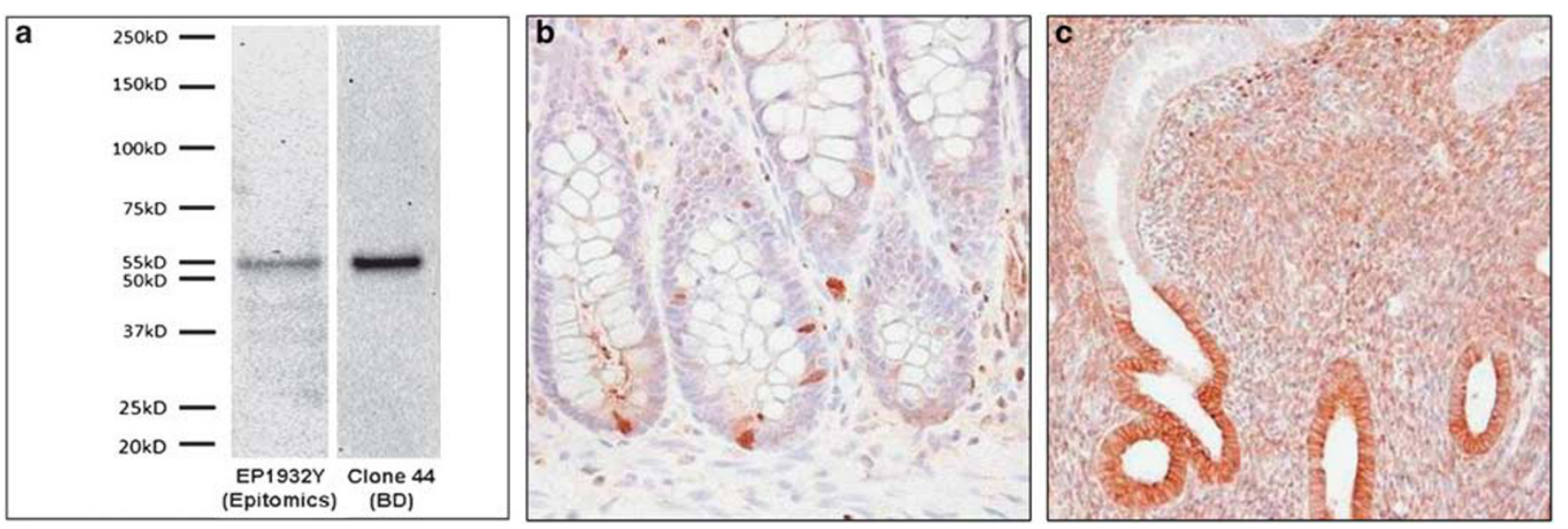

Figure 1 Specificity of ALDH1A1 antibodies. (a) Western blot performed using Epitomics and BD Biosciences antibodies on HHL-6 human hepatocyte cell lysate shows a robust $55 \mathrm{kDa}$ band corresponding to the ALDH1A1 protein. (b) ALDH1A1 immunoreactivity in discrete staining of single cells at colon crypt bases. (c) Strong ALDH1A1 staining in deep portions of endometrium stratum basalis. 
discrete single cells at the colonic crypt bases, where intestinal stem cells are known to reside (Figure 1b). In endometrial glands, more diffuse immunoreactivity was observed, although localized to the deep portions of glands in the stratum basalis (Figure 1c). Endometrial stromal cells were also positive for ALDH1A1. Patterns of staining were comparable between the two ALDH1A1 antibodies, with slightly higher background staining observed with the BD Biosciences antibody. Thus, the results in the present study have been obtained mainly using the Epitomics antibody.

In normal tubal epithelial cells, ALDH1A1 staining was detected in all 29 cases. The staining pattern was always patchy and discontinuous, and in some areas strong staining marked discrete single cells, including both secretory and ciliated cell types (Figures 2a-d); however, more often, long stretches of epithelium showed diffuse immunoreactivity. There were no clear differences in the percentage of ALDH1A1-positive cells observed in sections of fimbriae compared with more proximal regions of the tube (distal fimbria: $46 \pm 29 \%$ vs ampulla cross sections: $42 \pm 29 \%, P=0.642$ ). As in endometrium, prominent staining was observed in stromal cells.

Recent work based on a mouse model has identified ALDH1A1-positive stem cells enriched at the ovarian hilum. ${ }^{16}$ In the mouse, this region represents the transition from mesothelium to tubaltype epithelium; thus, presumably analogous to the tubal-mesothelial junctions present in the human fallopian tube fimbria. In our cohort, ALDH1A1positive cells were detected in $15(54 \%)$ of 28 junctions (Figures 2e and f). ALDH1A1 expression was detected in ovarian surface epithelium in all eight ovaries. However, no consistent differences were observed at the hilar region or tubal-mesothelial junctions compared with normal ovarian surface epithelium or fallopian tube epithelium located elsewhere. Of note, evaluation of the ovarian surface epithelium revealed heterogeneous ALDH1A1 staining, which occurred more often in cells with cuboidal or columnar morphology, rather than in flat mesothelial cells (Figures 3a-d). These areas frequently demonstrate strong calretinin positivity, consistent with mesothelial differentiation, along with Pax-8 nuclear staining. Pax-8 expression in ovarian surface epithelium has been previously reported, and, while controversial, has been suggested to represent Müllerian metaplasia. ${ }^{20}$ Interestingly, diffuse and intense ALDH1A1 immunoreactivity was observed in all rete ovarii (Figures $3 \mathrm{e}$ and $\mathrm{f}$ ) and foci of endosalpingiosis examined.

Next, we assessed ALDH1A1 expression in p53 signatures, serous tubal intraepithelial carcinoma, and high-grade serous carcinoma (Figure 4). A total of 11 p53 signatures, morphologically normal tubal epithelial cells with intense p53 staining, were identified; all were ALDH1A1 negative. ALDH1A1 staining was detected in 0 of 17 tubal intraepithelial lesions and in $41(61 \%)$ of 67 carcinomas. Eleven of the 17 fallopian tubes with serous tubal intraepithelial carcinoma also had invasive malignancy on the same tissue section, thus enabling direct comparison of their respective staining characteristics. Staining patterns were generally consistent between matched intraepithelial and invasive components, with the exception of two cases, in which focal immunoreactivity was detected in high-grade serous carcinoma, but not within serous tubal intraepithelial carcinoma. Although ALDH1A1 expression was detected to some degree in many high-grade serous carcinomas, in most cases, only rare ALDH1A1positive cells were observed (Figure 5a). In our cohort of 26 paired primary and recurrent ovarian serous carcinomas, comparable frequencies of ALDH1A1-positive tumor cells were observed between matched tumor pairs (mean \pm s.d.: $8.0 \pm 14.6 \%$ vs $4.5 \pm 7.7 \%, \quad P=0.28$ ). There was a significant decrease in the percentage of ALDH1A1-stained cells in serous tubal intraepithelial carcinoma and p53 signatures as compared with normal fallopian tubes $(P<0.0001)$. The percentages of ALDH1A1positive cells in different tissues and lesions are summarized in Figure 6.

To validate our immunohistochemistry results, representative cases were stained using the clone 44/BD Biosciences ALDH1A1 antibody. The performance of both antibodies was comparable, with no appreciable differences in the distribution of staining and the percentage of ALDH1A1-positive cells. In silico analysis of TCGA mRNA expression data for ovarian serous carcinoma ${ }^{21}$ revealed decreased expression in tumor relative to normal fallopian tube (tumor/normal expression ratio $=0.13, P<0.0001$ ). Moreover, ALDH1A1 expression level in primary ovarian cancer was not associated with overall survival $(\mathrm{HR}=1.04, P=0.757$; Figure $5 \mathrm{~b})$.

\section{Discussion}

ALDH1A1 has been proposed as a marker for cancer stem cells or cancer initiating cells in various types of human cancer, including ovarian carcinoma. Previous experimental work demonstrated ALDH1A1-positive ovarian cancer cells to be more tumorigenic than ALDH1A1-negative cells. ${ }^{22,23}$ It is therefore plausible that these ALDH1A1-expressing tumor cells may be derived from somatic stem cells, likely from the fallopian tube. Indeed, the mouse ovarian hilum has recently been shown to be enriched for ALDH1A1-positive stem cells with increased susceptibility to malignant transformation. ${ }^{16}$ The purpose of this study was to comprehensively characterize the expression of ALDH1A1 in normal human fallopian tube, tubal-mesothelial junctions, ovarian surface epithelium, and in lesions thought to represent different stages of tumor progression - namely, p53 signatures, serous tubal intraepithelial carcinoma, and primary and recurrent high-grade serous carcinomas. 

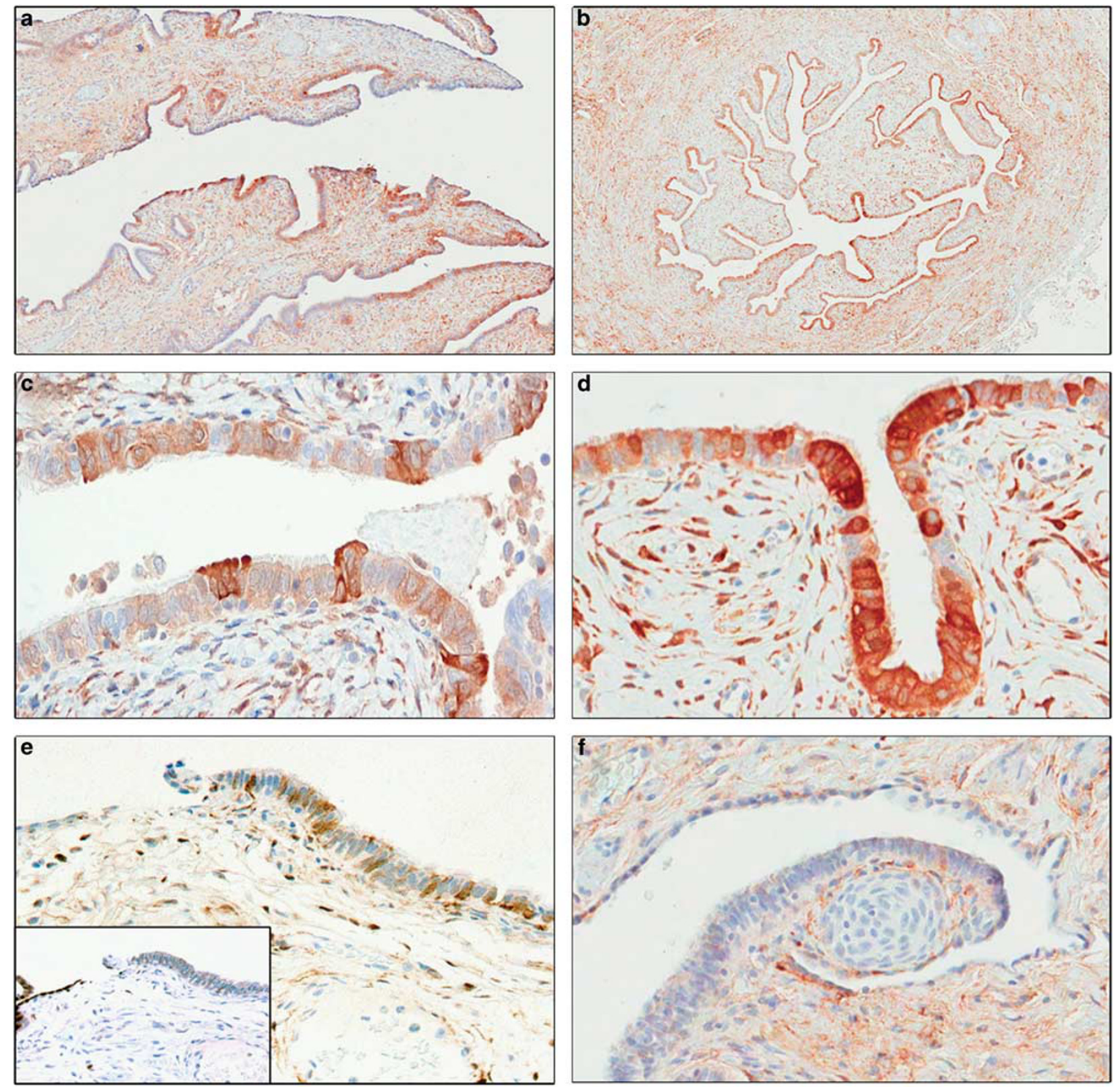

Figure 2 ALDH1A1 staining in normal fallopian tube. Sections from (a) distal fimbriae and (b) ampulla demonstrating similar staining patterns. (c, d) High magnification reveals ALDH1A1 to be variably expressed in secretory and ciliated epithelial cells. (e) Tubalmesothelial junction, representing the transition from mesothelium to tubal epithelium (calretinin stain, inset); ALDH1A1 staining is positive in epithelial cells in this case. (f) Another tubal-mesothelial junction with focus of transitional metaplasia; negative for ALDH1A1. (a-f) ALDH1A1 immunohistochemical stain.

Somatic stem cells are self-renewing cells that give rise to the differentiated cells necessary for tissue function. They are usually present in small numbers and tend to be located in a discrete anatomic niche such as the colonic crypts and hair follicle bulbs. It is unlikely that the ALDH1A1positive tubal cells, described in this study, are tubal stem cells as they correspond to ciliated and secretory cells, which are terminally differentiated. Similar staining patterns have been observed in another recent study evaluating ALDH1A1 expression in normal fallopian tube using a polyclonal goat antibody (L15; Santa Cruz Biotechnology). ${ }^{24}$ Consistent with our results, many positively stained cells were seen in the tubal fimbriae and ampulla. However, in contrast to that report demonstrating ALDH1A1 expression to be largely confined to secretory cells, we observed ALDH1A1 immunoreactivity in both secretory and ciliated epithelial cells. Regardless, our findings cast doubt on ALDH1A1 as a specific 

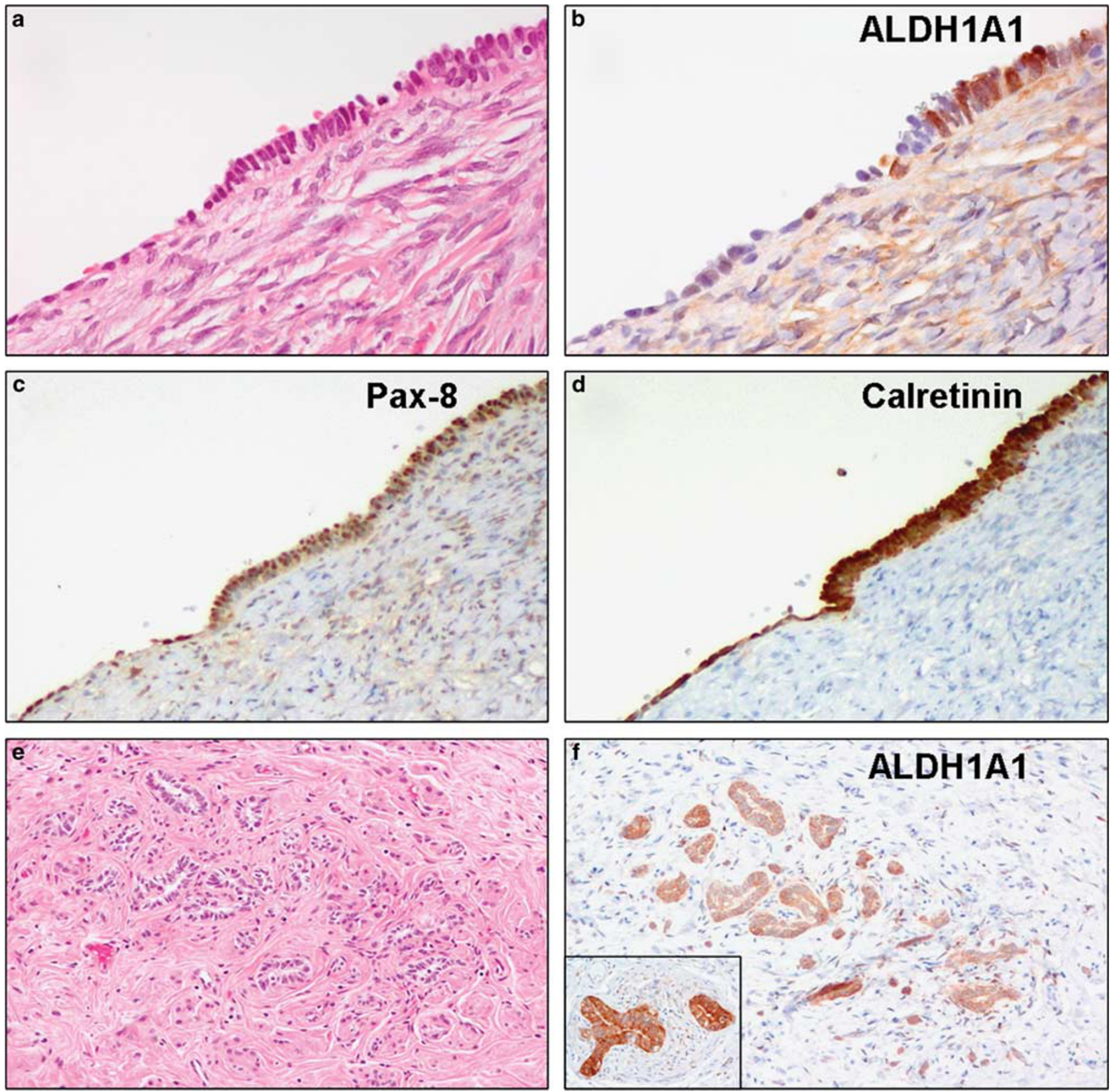

Figure 3 ALDH1A1 staining in normal ovary. (a) Ovarian surface lined by flat and columnar epithelium (hematoxylin and eosin stain). (b) There is heterogeneous staining of ovarian surface epithelium, preferentially in cells with columnar morphology. (ALDH1A1 immunohistochemical stain). The same area demonstrates positivity for (c) Pax-8, as well as (d) calretinin. (e) Rete ovarii (hematoxylin and eosin stain) with (f) diffuse and strong ALDH1A1 expression (high magnification, inset).

marker for somatic stem cells in human fallopian tube. ALDH1A1 encodes an enzyme belonging to the ALDH family that catalyzes the oxidation (dehydrogenation) of aldehydes, which in the fallopian tube may have a role in facilitating the transport of blastocysts. $^{25}$ The finding of diffuse ALDH1A1 positivity within foci of endosalpingiosis is consistent with their presumed origin from fallopian tube epithelium, ${ }^{1}$ whereas the significance of ALDH1A1 staining of rete ovarii, derived from mesonephric remnants, is unknown.
The most interesting observation in this study is that ALDH1A1 expression is completely absent in serous tubal intraepithelial carcinoma, the putative precursor of high-grade serous carcinoma. This finding, along with the observation that all evaluated p53 signatures were also ALDH1A1 negative, suggests that loss of ALDH1A1 expression is an early event in the pathogenesis of this malignancy. The cell of origin of high-grade serous carcinoma is therefore either a cell that is intrinsically ALDH1A1 negative or an ALDH1A1-positive cell in which 
p53 signature

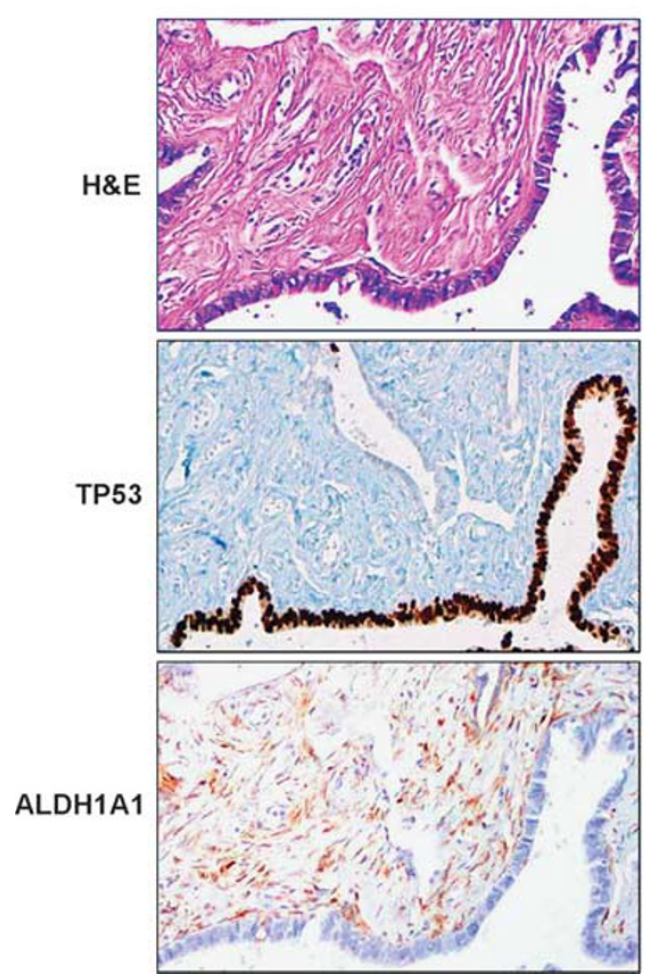

Serous tubal intraepithelial carcinoma
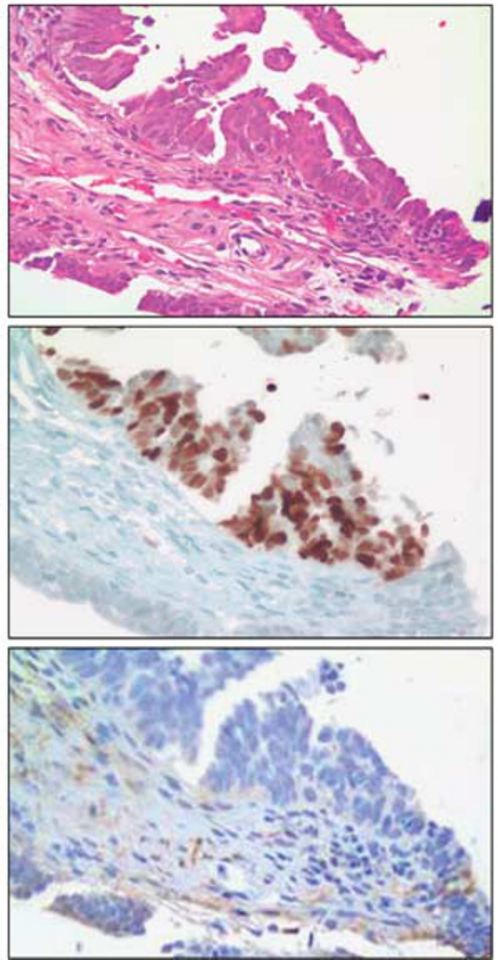

Serous tubal intraepithelial carcinoma with invasive serous carcinoma

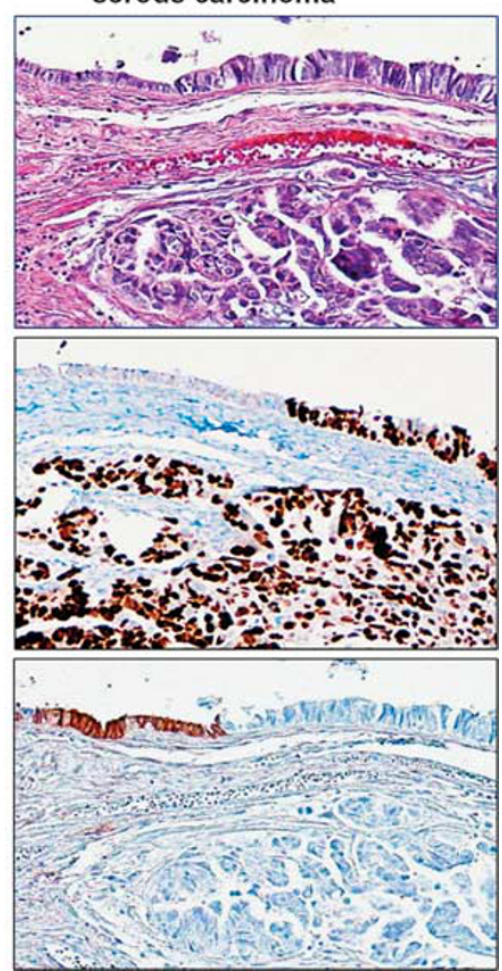

Figure 4 ALDH1A1 staining in tubal lesions. 'p53 signature,' referring to morphologically normal tubal epithelium with intense nuclear staining for TP53 protein, shows completely negative ALDH1A1 immunoreactivity (left panel). Loss of ALDH1A1 in serous tubal intraepithelial carcinoma (middle panel). In another patient, serous tubal intraepithelial carcinoma and invasive high-grade serous carcinoma showing complete absence of ALDH1A1, while adjacent normal epithelium is intensely positive (right panel).

expression of this enzyme has been suppressed during neoplastic transformation. Previous work suggests that silencing of ALDH1A1 may be mediated by the histone methyltransferase, EZH2, that is upregulated in high-grade serous carcinoma. Knockdown of EZH2 resulted in upregulation of ALDH1A1 in the SKOV3 cell line, ${ }^{26}$ in which ALDH1A1 expression is otherwise suppressed. Of course, there are alternative explanations for the absence of ALDH1A1 expression in precursor lesions. For example, it remains a possibility that the complete absence of ALDH1A1-positive cells in serous tubal intraepithelial carcinoma or p53 signatures may be biased by the relatively limited number of cells assessed, given the small size of these lesions.

We also observed considerably decreased ALDH1A1 staining in high-grade serous carcinoma compared to normal tubal epithelium, confirming in silico analysis of TCGA expression data. Accordingly, ALDH1A1 expression in this tumor type is probably of minimal biological significance, as staining was often limited to a few cells in the positive cases and likely reflects intratumoral heterogeneity in gene expression. ${ }^{27}$ Nevertheless, we cannot exclude the possibility that the ALDH1A1-positive subpopulation may be enriched for cancer stem cells, as suggested in previous work..$^{22,23,28}$ If this were the case, one must conclude that either highgrade serous carcinoma does not develop from fallopian tube epithelium, where ALDH1A1 does not appear to label stem cells, or ALDH1A1 expression has different functions in malignant vs normal Müllerian epithelium. That is to say, in the normal fallopian tube, ALDH1A1 participates in aldehyde metabolism, and not maintaining 'stemness,' whereas in serous carcinoma ALDH1A1 expression is involved in the development of cancer stem cells. This scenario, however, is difficult to reconcile with the absence of ALDH1A1 expression in serous tubal intraepithelial carcinoma.

Furthermore, it should be recognized that results from previous experimental work have not always been consistent. Stewart et $a l^{28}$ sorted primary patient-derived serous ovarian carcinoma cells using a variety of putative cancer stem cell markers, including ALDH enzymatic activity, and subsequently assayed their ability to form tumors in immunodeficient mice. Although the frequency of tumor-initiating cells was generally higher in the $\mathrm{ALDH}^{\text {hi }}$ subpopulation (except for one patient, from which all tumor-initiating cells were $\mathrm{ALDH}^{\mathrm{lo}}$ ), the absolute number was comparable between ALDH ${ }^{\text {hi }}$ 

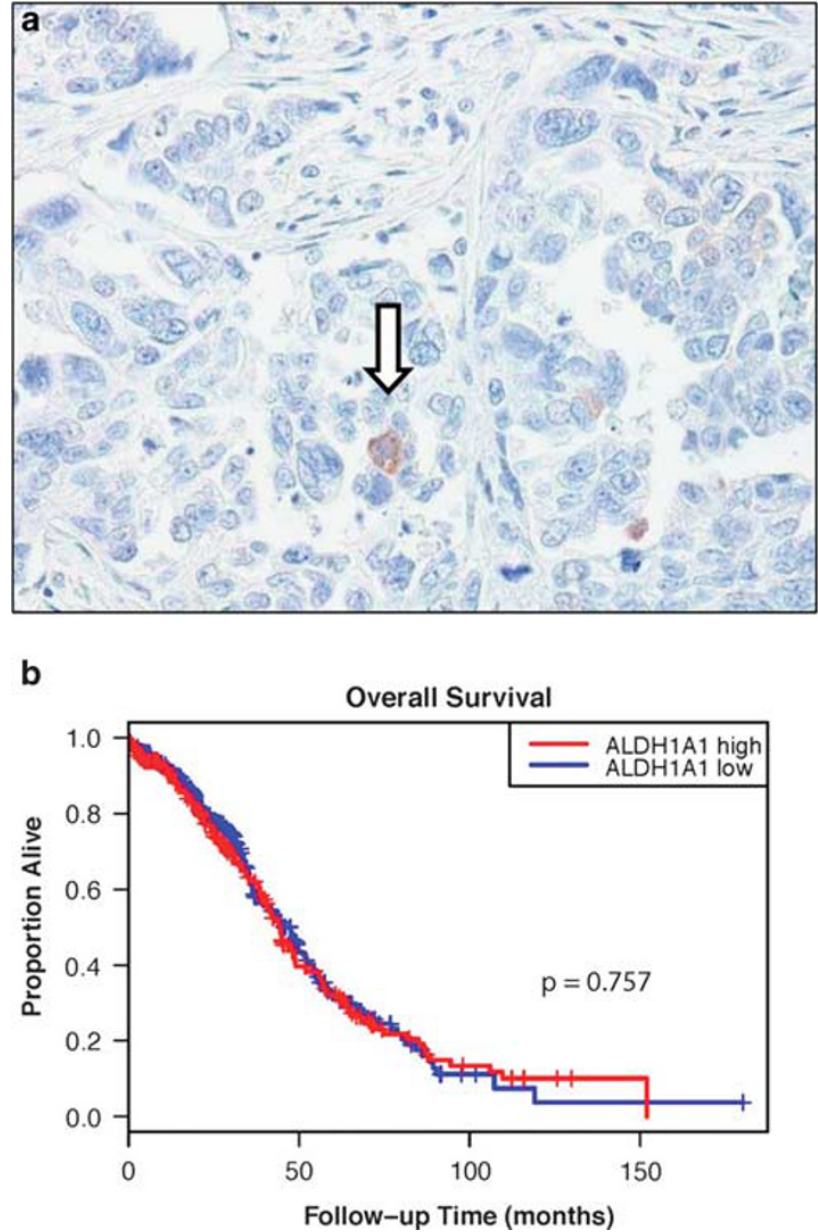

Figure 5 ALDH1A1 expression in high-grade serous carcinoma. (a) High-grade serous carcinoma with ALDH1A1 staining restricted to rare tumor cells (arrow). (b) No difference in overall survival when stratified by ALDH1A1 expression level (split at median), based on in silico analysis of TCGA mRNA expression data set.

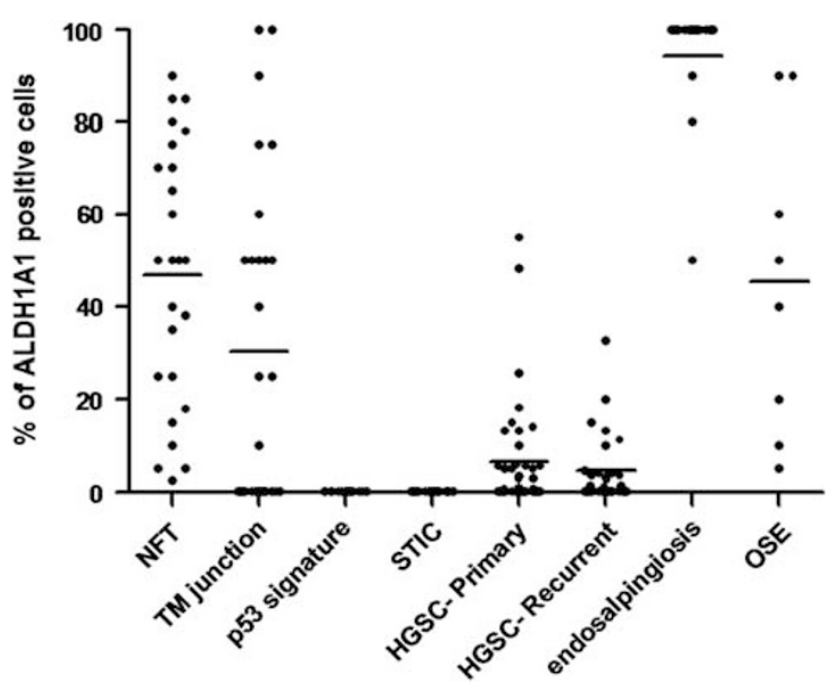

Figure 6 The percentage of ALDH1A1-positive cells in different types of tissues/lesions. Each dot represents an individual sample with horizontal lines representing the mean for each group. and $\mathrm{ALDH}^{\mathrm{lo}}$ fractions, demonstrating the poor specificity of this marker. Rather than suggesting an association between ALDH1A1 and cancer stem cell identity, an alternative explanation may be that the ability to metabolize aldehydes somehow confers a survival advantage to human ovarian cancer cells transplanted into a xenogeneic environment.

While several clinicopathological studies have suggested ALDH1A1 expression to correlate with poor prognosis in high-grade serous ovarian carcinoma, ${ }^{22,23,29,30}$ one study found no significant association. ${ }^{26}$ Similarly, our in silico analysis of the TCGA mRNA expression data set failed to reveal any prognostic significance, and, furthermore, we did not observe a significant difference in ALDH1A1expressing cells between primary and recurrent serous ovarian tumors.

In summary, we demonstrate that ALDH1A1expressing epithelial cells are abundant in the normal fallopian tube, but not in p53 signatures or serous tubal intraepithelial carcinoma. ALDH1A1positive cells can occasionally be observed in some high-grade serous carcinomas, but usually restricted to only a few tumor cells. These findings suggest that ALDH1A1 is probably not a specific marker for fallopian tube stem cells, and demonstrate that its loss of expression is an early event in the development of high-grade serous carcinoma. Identification of more specific markers of Müllerian epithelial stem cells is necessary to identify the putative somatic stem cell population involved in the pathogenesis of this malignancy.

\section{Acknowledgments}

This study was supported by a grant from US Department of Defense W81XWH-11-2-0230/ OC100517.

\section{Disclosure/conflict of interest}

The authors declare no conflict of interest.

\section{References}

1 Kurman RJ, Shih Ie M. Molecular pathogenesis and extraovarian origin of epithelial ovarian cancer-shifting the paradigm. Hum Pathol 2011;42:918-931.

2 Colgan TJ, Murphy J, Cole DE, et al. Occult carcinoma in prophylactic oophorectomy specimens: prevalence and association with BRCA germline mutation status. Am J Surg Pathol 2001;25:1283-1289.

3 Medeiros F, Muto MG, Lee Y, et al. The tubal fimbria is a preferred site for early adenocarcinoma in women with familial ovarian cancer syndrome. Am J Surg Pathol 2006;30:230-236.

4 Kindelberger DW, Lee Y, Miron A, et al. Intraepithelial carcinoma of the fimbria and pelvic serous carcinoma: Evidence for a causal relationship. Am J Surg Pathol 2007;31:161-169. 
5 Lee Y, Miron A, Drapkin R, et al. A candidate precursor to serous carcinoma that originates in the distal fallopian tube. J Pathol 2007;211:26-35.

6 Visvader JE. Cells of origin in cancer. Nature 2011; 469:314-322.

7 Chui MH. Insights into cancer metastasis from a clinicopathologic perspective: epithelial-mesenchymal transition is not a necessary step. Int J Cancer 2013; 132:1487-1495.

8 O'Brien CA, Pollett A, Gallinger S, et al. A human colon cancer cell capable of initiating tumour growth in immunodeficient mice. Nature 2007;445:106-110.

9 Douville J, Beaulieu R, Balicki D. ALDH1 as a functional marker of cancer stem and progenitor cells. Stem Cells Dev 2009;18:17-25.

10 Chute JP, Muramoto GG, Whitesides J, et al. Inhibition of aldehyde dehydrogenase and retinoid signaling induces the expansion of human hematopoietic stem cells. Proc Natl Acad Sci USA 2006;103:11707-11712.

11 Ginestier C, Hur MH, Charafe-Jauffret E, et al. ALDH1 is a marker of normal and malignant human mammary stem cells and a predictor of poor clinical outcome. Cell Stem Cell 2007;1:555-567.

12 Jimeno A, Feldmann G, Suarez-Gauthier A, et al. A direct pancreatic cancer xenograft model as a platform for cancer stem cell therapeutic development. Mol Cancer Ther 2009;8:310-314.

13 Hellsten R, Johansson M, Dahlman A, et al. Galiellalactone inhibits stem cell-like ALDH-positive prostate cancer cells. PLoS ONE 2011;6:11.

14 Ma S, Chan KW, Lee TK, et al. Aldehyde dehydrogenase discriminates the CD133 liver cancer stem cell populations. Mol Cancer Res 2008;6:1146-1153.

15 Clay MR, Tabor M, Owen JH, et al. Single-marker identification of head and neck squamous cell carcinoma cancer stem cells with aldehyde dehydrogenase. Head Neck 2010;32:1195-1201.

16 Flesken-Nikitin A, Hwang CI, Cheng CY, et al. Ovarian surface epithelium at the junction area contains a cancer-prone stem cell niche. Nature 2013;495: 241-245.

17 Seidman JD, Yemelyanova A, Zaino RJ, et al. The fallopian tube-peritoneal junction: a potential site of carcinogenesis. Int J Gynecol Pathol 2011;30:4-11.

18 Visvanathan K, Vang R, Shaw P, et al. Diagnosis of serous tubal intraepithelial carcinoma based on morphologic and immunohistochemical features: a reproducibility study. Am J Surg Pathol 2011;35: 1766-1775.

19 Vang R, Visvanathan K, Gross A, et al. Validation of an algorithm for the diagnosis of serous tubal intraepithelial carcinoma. Int J Gynecol Pathol 2012;31:243-253.

20 Auersperg N. The origin of ovarian carcinomas: a unifying hypothesis. Int J Gynecol Pathol 2011;30: 12-21.

21 Cancer Genome Atlas Research Network. Integrated genomic analyses of ovarian carcinoma. Nature 2011; 474:609-615.

22 Wang YC, Yo YT, Lee HY, et al. ALDH1-bright epithelial ovarian cancer cells are associated with CD44 expression, drug resistance, and poor clinical outcome. Am J Pathol 2012;180:1159-1169.

23 Landen CN Jr., Goodman B, Katre AA, et al. Targeting aldehyde dehydrogenase cancer stem cells in ovarian cancer. Mol Cancer Ther 2010;9:3186-3199.

24 Auersperg N. The stem-cell profile of ovarian surface epithelium is reproduced in the oviductal fimbriae, with increased stem-cell marker density in distal parts of the fimbriae. Int J Gynecol Pathol 2013;32:444-453.

25 Vermot J, Fraulob V, Dolle P, et al. Expression of enzymes synthesizing (aldehyde dehydrogenase 1 and reinaldehyde dehydrogenase 2) and metabolizaing (Cyp26) retinoic acid in the mouse female reproductive system. Endocrinology 2000;141:3638-3645.

$26 \mathrm{Li} \mathrm{H}$, Bitler BG, Vathipadiekal V, et al. ALDH1A1 is a novel EZH2 target gene in epithelial ovarian cancer identified by genome-wide approaches. Cancer Prev Res (Phila) 2012;5:484-491.

27 Tarin D. Inappropriate gene expression in human cancer and its far-reaching biological and clinical significance. Cancer Metastasis Rev 2012;31:21-39.

28 Stewart JM, Shaw PA, Gedye C, et al. Phenotypic heterogeneity and instability of human ovarian tumorinitiating cells. Proc Natl Acad Sci USA 2011;108: 6468-6473.

29 Liebscher CA, Prinzler J, Sinn BV, et al. Aldehyde dehydrogenase 1/epidermal growth factor receptor coexpression is characteristic of a highly aggressive, poor-prognosis subgroup of high-grade serous ovarian carcinoma. Hum Pathol 2013;44:1465-1471.

30 Deng S, Yang X, Lassus $\mathrm{H}$, et al. Distinct expression levels and patterns of stem cell marker, aldehyde dehydrogenase isoform 1 (ALDH1), in human epithelial cancers. PLoS ONE 2010;5:e10277. 